\title{
Individual xenograft as a personalized therapeutic resort for women with metastatic triple-negative breast carcinoma
}

\author{
Guilhem Bousquet ${ }^{1,2,3^{*}}$, Jean-Paul Feugeas ${ }^{1,4}$, Irmine Ferreira ${ }^{1,2}$, Laetitia Vercellino ${ }^{1,5}$, Nathalie Jourdan ${ }^{6}$, \\ Philippe Bertheau ${ }^{1,2,7}$, Cédric de Bazelaire ${ }^{1,2,7}$, Emmanuel Barranger ${ }^{1,8}$ and Anne Janin ${ }^{1,2,7^{*}}$
}

\section{Findings}

A localized left breast ductal invasive triple-negative breast carcinoma (TNBC) was diagnosed in a 44-yearold woman. After surgery, she was treated with chemotherapy and radiation therapy in accordance with national guidelines. At the end of treatment, she had local and metastatic relapse with multiple sub-diaphragmatic lymph nodes.

With her written consent, imagery-guided biopsies of a metastatic lymph node were performed, one sample being used for genomic analyses and another for immediate xenograft (Additional file 1: Supplementary methods). Because of the early metastases after docetaxel and epirubicin-cyclophosphamide, she received cisplatin but the disease progressed after 3 months.

Meanwhile, the transcriptomic analysis of the metastatic sample enabled a molecular subclassification as Basal-Like 2 with EGFR mRNA overexpression [1,2]. We did not find any EGFR or K-RAS mutation. After successful engraftment of the metastastic samples, we tested eight different chemotherapies over 1 month on the xenograft model. The combination of paclitaxel and cetuximab was the most efficient (Figure 1A), so it was offered to the patient. After 3 months of this second-line treatment, the metabolic response was almost complete (Figure 1B, C).

In compliance with French bioethics law (\#2004-800, $06 / 08 / 2004)$, the patient had been informed of the research use of the part of her biopsy samples remaining after diagnosis had been established and did not oppose it. The treatment combining paclitaxel and cetuximab was discussed and approved in a multidisciplinary meeting and then offered to the patient. Informed written

\footnotetext{
* Correspondence: guilhem.bousquet@sls.aphp.fr; anne.janin728@gmail.com 1 Université Paris Diderot, Sorbonne Paris Cité, Laboratoire de Pathologie, UMR-S 1165, F-75010 Paris, France

Full list of author information is available at the end of the article
}

consent was obtained from the patient. The Clinical Research Board Ethics Committee (in French, 'Comité de Protection des Personnes') approved this study (CPP Ilede-France \#13218). The University Institute Board Ethics Committee for experimental animal studies approved this study (\#2012-15/728-0115).

The occurrence of metastases heavily affects prognosis for women with TNBC, with a median survival of under 15 months [3]. In addition, when progression occurs after first-line chemotherapy, there is no standard care.

Primary tumors are heterogeneous at the cellular and molecular level [4], and metastases derive from aggressive clones that have usually acquired resistance to firstline treatments. These tumor cells, which may be a minority in the primary tumor, are precisely those on which drug efficiency needs to be tested for individualized treatments for patients with metastases.

Recently, a molecular subclassification of TNBC was established, a new step toward innovative personalized treatments for patients with TNBC [1,2]. However, molecular analyses still have limitations, since molecular biomarkers are integrated into complex pathways and can rarely be directly used in daily oncologic practice. These limitations make it necessary to implement a different, complementary method. Individual xenografts from metastatic samples of TNBC are an additional, innovative tool and are more physiological than genomic analysis, as the tumor is in a mammalian environment and can be followed up over time. In pancreatic cancer, which shares poor prognosis and high metastatic potential with TNBC, individual xenografts have been proposed in a pilot study for personalized treatments [5]. Individual xenografts could also be proposed as a personalized therapeutic resort for women with metastatic TNBC. 

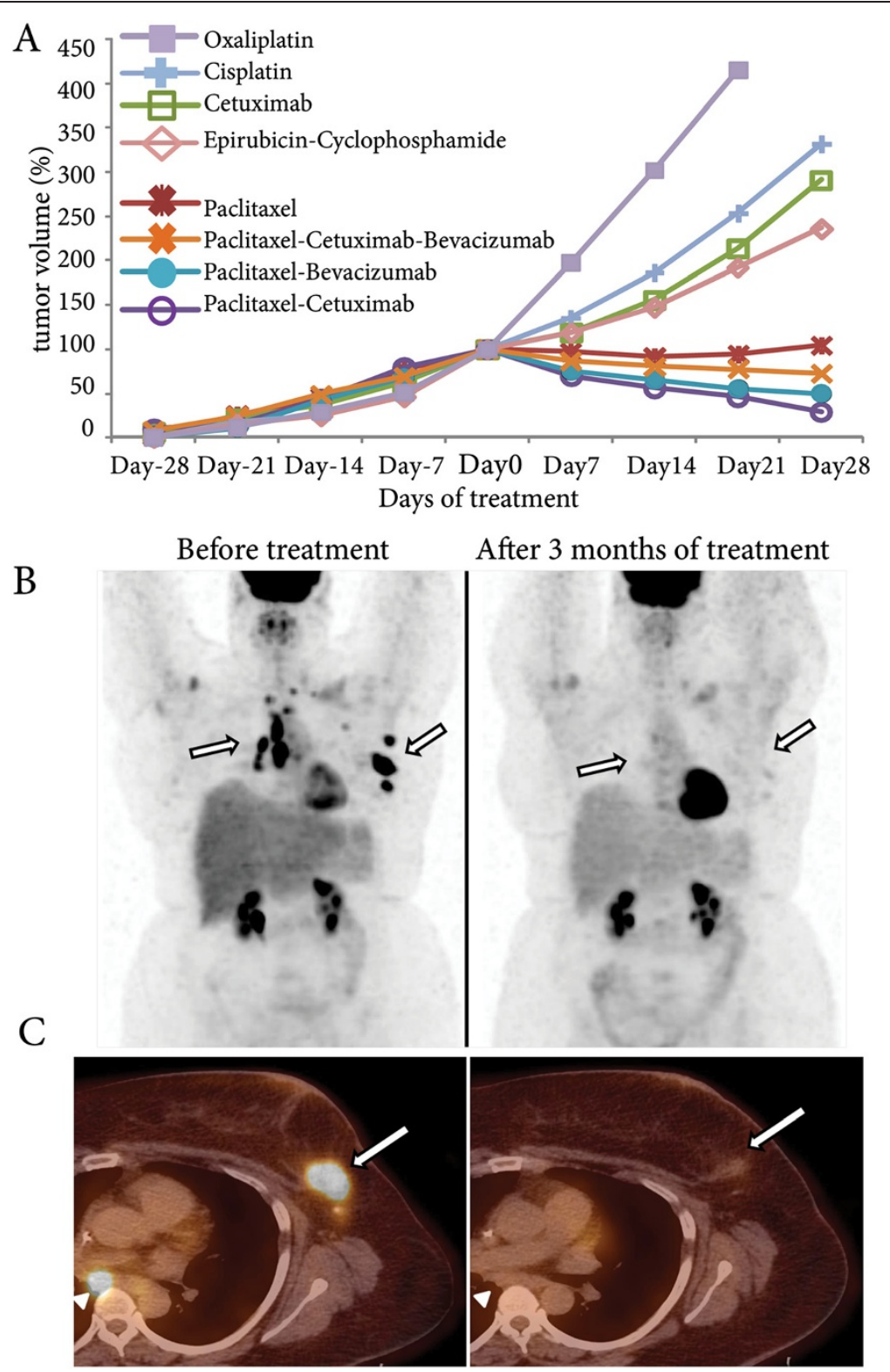

Figure 1 Individual xenograft efficiently guided treatment of a patient with metastatic triple-negative breast carcinoma. (A) In vivo effects of drugs or combination of drugs in the xenograft model obtained from metastatic triple-negative breast carcinoma. Mice $(n=5$ per group of treatment) were treated with drugs or combinations of drugs. Tumors were measured every week, from 4 weeks before the treatment started to 4 weeks after treatment. Tumor volumes were calculated at each time-point. The greatest tumor growth inhibition is observed with the combination of paclitaxel and cetuximab (purple circle). (B) Positron emission tomography (PET), maximum intensity projection. The left panel shows baseline PET with intense fluoro-deoxyglucose (FDG) uptake in sub-diaphragmatic breast and lymph node lesions (white arrows); the right panel shows PET after two cycles of combined paclitaxel and cetuximab. There is a marked decrease (88\%) of SUV $\max$ (maximum standardized uptake value within the region of interest) in target lesions (white arrows) and thus a partial metabolic response according to PERCIST (PET Response Criteria in Solid Tumors) criteria. (C) Fused PET/computed tomography. The left panel shows an image at baseline with intense FDG uptake in a left breast lesion (SUV $\max =17.2$, white arrow) and in a subcarinar lymph node (SUV $\max =14.3$, white arrowhead); after two treatment cycles (right panel), SUV $V_{\max }$ of both target lesions (white arrow, white arrowhead) is below background level. 


\section{Additional file}

Additional file 1: Supplementary methods.

\section{Abbreviation}

TNBC: Triple-negative breast carcinoma.

\section{Competing interests}

The authors declare that they have no competing interests.

\section{Authors' contributions}

GB conceived and designed the study, provided the study material or patient, collected and assembled the data, provided data analysis and interpretation, and drafted the manuscript. J-PF provided data analysis and interpretation, and performed the statistical analyses. IF collected and assembled data and performed the xenografts. LV collected and assembled data, provided data analysis and interpretation, and performed the positron emission tomography/computed tomography. NJ provided data analysis and interpretation. PB provided the study material. CdB provided the study material or patient and performed tumor biopsies. EB provided the study material or patient. AJ conceived and designed the study, located financial support, provided administrative support, provided the study material or patient, collected and assembled data, provided data analysis and interpretation, and drafted the manuscript. All authors read and approved the final manuscript.

\section{Acknowledgments}

This work was supported by Région Île-de-France, Institut National du Cancer (INCa). We thank Angela Swaine for the English language review. We thank the patient for her consent to the publication of this study.

\section{Author details}

'Université Paris Diderot, Sorbonne Paris Cité, Laboratoire de Pathologie, UMR-S 1165, F-75010 Paris, France. ' INSERM, U1165-Paris, F-75010 Paris, France. ${ }^{3}$ AP-HP-Hôpital Saint-Louis, Service d'Oncologie-Paris, F-75010 Paris, France. ${ }^{4}$ INSERM, U722-Paris, F-75010 Paris, France. ${ }^{5}$ AP-HP-Hôpital Saint-Louis, Service de Médecine Nucléaire-Paris, F-75010 Paris, France. ${ }^{6}$ AP-HP-Hôpital Saint-Louis, Pharmacie-Paris, F-75010 Paris, France.

${ }^{7}$ AP-HP-Hôpital Saint-Louis, Service de Pathologie-Paris, F-75010 Paris, France. ${ }^{8}$ Centre Antoine Lacassagne, Service de Chirurgie-Gynécologie - Nice, F-06189 Paris, France.

Published: 11 Feb 2014

\section{References}

1. Lehmann BD, Bauer JA, Chen X, Sanders ME, Chakravarthy AB, Shyr Y, Pietenpol JA: Identification of human triple-negative breast cancer subtypes and preclinical models for selection of targeted therapies. J Clin Invest 2011, 121:2750-2767.

2. Masuda H, Baggerly KA, Wang Y, Zhang Y, Gonzalez-Angulo AM, Meric-Bernstam F, Valero V, Lehmann BD, Pietenpol JA, Hortobagyi GN, Symmans WF, Ueno NT: Differential response to neoadjuvant chemotherapy among 7 triple-negative breast cancer molecular subtypes. Clin Cancer Res 2013, 19:5533-5540.

3. Kassam F, Enright K, Dent R, Dranitsaris G, Myers J, Flynn C, Fralick M, Kumar $R$, Clemons M: Survival outcomes for patients with metastatic triplenegative breast cancer: implications for clinical practice and trial design. Clin Breast Cancer 2009, 9:29-33.

4. Gerlinger M, Rowan AJ, Horswell S, Larkin J, Endesfelder D, Gronroos E, Martinez P, Matthews N, Stewart A, Tarpey P, Varela I, Phillimore B, Begum S, McDonald NQ, Butler A, Jones D, Raine K, Latimer C, Santos CR, Nohadani M, Eklund AC, Spencer-Dene B, Clark G, Pickering L, Stamp G, Gore M, Szallasi Z, Downward J, Futreal PA, Swanton C: Intratumor heterogeneity and branched evolution revealed by multiregion sequencing. N Engl J Med 2012, 366:883-892.

5. Hidalgo M, Bruckheimer E, Rajeshkumar NV, Garrido-Laguna I, De Oliveira E, Rubio-Viqueira B, Strawn S, Wick MJ, Martell J, Sidransky D: A pilot clinical study of treatment guided by personalized tumorgrafts in patients with advanced cancer. Mol Cancer Ther 2011, 10:1311-1316.

\section{$10.1186 / \mathrm{bcr} 3615$}

Cite this article as: Bousquet et al.: Individual xenograft as a personalized therapeutic resort for women with metastatic triplenegative breast carcinoma. Breast Cancer Research 2014, 16:401 